\title{
Cross-modal extinction in a boy with severely autistic behavior and high verbal intelligence
}

\author{
Corresponding Author: Dr. Yoram Bonneh, PhD \\ Corresponding Author's Institution: University of Haifa \& The Weizmann Institute of Science
}

Authors:

Yoram S Bonneh, University of Haifa, Haifa, Israel \& The Weizmann Institure of Science, Rehovot, Israel Matthew K Belmonte, Department of Human Development, Cornell University, Ithaca, NY

Francesca Pei, , Department of Physiology and Biochemistry \& AISV, University of Pisa, Italy

Portia E Iversen, Cure Autism Now, Los-Angeles, CA

Tal Kenet, Department of Neurology, Massachusetts General Hospital, Boston, MA

Natacha Akshoomoff, Department of Psychiatry, School of Medicine, University of California, San Diego

Yael Adini, The Weizmann Institure of Science, Rehovot, Israel

Helen J Simon, Smith-Kettlewell Eye Research Inst., San Francisco, CA

Christopher I Moore, Department of Brain and Cognitive Sciences, Cambridge, Massachusetts Institute of Technology, MA

John F Houde, Department of Otolarygology, University of California, San Francisco, CA

Michael M Merzenich, Keck Center for Integrative Neuroscience, University of California, San Francisco, CA

Short title: Cross-modal extinction in autism

\section{Address for correspondence:}

Dr. Yoram Bonneh

Department of Neurobiology, The Weizmann Institute of Science

Rehovot 76100, Israel

email: Yoram.Bonneh@weizmann.ac.il

Tel: +972 544995277

Fax: +972 8 934-4131 


\title{
Cross-modal extinction in a boy with severely autistic behavior and high verbal intelligence
}

\begin{abstract}
Anecdotal reports from individuals with autism suggest a loss of awareness to stimuli from one modality in the presence of stimuli from another. Here we document such a case in a detailed study of T.M., a 13-year-old boy with autism in whom significant autistic behaviors are combined with an uneven IQ profile of superior verbal and low performance abilities. Although T.M.'s speech is often unintelligible and his behavior is dominated by motor stereotypies and impulsivity, he can communicate by typing or pointing independently within a letter board. A series of experiments using simple and highly salient visual, auditory, and tactile stimuli demonstrated a hierarchy of cross-modal extinction, in which auditory information extinguished other modalities at various levels of processing. T.M. also showed deficits in shifting and sustaining attention. These results provide evidence for mono-channel perception in autism and suggest a general pattern of winner-takes-all processing in which a stronger stimulus-driven representation dominates behavior, extinguishing weaker representations.
\end{abstract}

Keywords: autism; extinction; perception; attention; cross-modal; multimodal; auditory; visual; tactile 


\section{Introduction}

Several first-person reports of autism (Grandin, 1995; Lawson, 2003; D. Williams, 1996) describe autistic perception as a "mono channel" system in which only one source of input at a time can be processed. For example, attending to speech may cause a complete loss of tactile awareness, and even within a single modality, attending to intonation may cause loss of awareness of words. This unimodal style of perception may be related to the finding of stimulus over-selectivity (Lovaas, Koegel, \& Schreibman, 1979), where people with autism classify complex, multimodal stimuli using only a unimodal criterion, to the idea of "monotropism" which characterizes autism as a tightly focused attention style (Murray, Lesser, \& Lawson, 2005) and also to the many findings of impairment in shifting attention in autism (reviewed in (Allen \& Courchesne, 2001)). Here we report on T.M., a 13-year-old boy with autism presenting with complaints of severe impairment in multisensory perception (“when I hear, my vision shuts down”).

Most cognitive studies of autism include only "high-functioning” individuals who can speak and who can comply with experimental paradigms, and thus omit the very people in whom autism's effects are most severe. T.M. shows many of the symptoms typical of "lowfunctioning” cases - including stereotyped movements, repetitive behaviors, inability to produce readily intelligible speech, lack of eye contact, and hyposensitivity to proprioception, touch and pain - yet can communicate independently using a keyboard, a letter board, or even handwriting, and can understand and perform complex psychophysical experiments despite his intrusive behaviors. This combination of typical severe autistic symptoms with an unusual communicative ability makes T.M. a valuable window onto the very large population of "low-functioning" individuals with autism who may otherwise escape the attention of cognitive neuroscientists.

T.M.'s strong ability to communicate enabled us to test experimentally the reports of his cross-modal interference, and to identify possible abnormalities in his sensory information processing. Cross-modal interference may reflect a difficulty in dividing attention between modalities typically occurring under conditions of high attentional load and low stimulus 
salience. Alternatively, it may reflect an explicit loss of awareness, of the type found in hemi-neglect patients (Driver \& Vuilleumier, 2001), which affects even highly salient stimuli without any attentional load. To test these possibilities and their functional consequences, we used basic stimuli and tasks that are known to involve minimal attentional load, sometimes called "preattentive" (Braun \& Julesz, 1998), e.g., the detection of highly salient sounds and visual patches. We report four psychophysical experiments at a range of processing levels that provide ample evidence for severe cross-modal interference and consequently a failure to integrate perceptual information. We also report on a unimodal visual experiment that replicates in this severely autistic subject the finding of slowed shifting of attention and identifies a deficit in sustained attention. Finally, we interpret these results in terms of a general pattern of abnormal neural information processing in autism.

\section{Case details}

Information obtained from clinical interviews, detailed diagnostic testing, and clinical judgment indicated that T.M. met DSM-IV criteria for autistic disorder. The consensus of the three experienced clinicians who evaluated him (LW, JG, BH) was that he appeared to be similar in many ways to those individuals with autism first described by Kanner (1943). The results from the Autism Diagnostic Interview-Revised (Lord, Rutter, \& Le Couteur, 1994), administered to his mother by a psychologist specializing in autism diagnosis, were above the autism cut-off and consistent with a classification of autism. Module 1 of the Autism Diagnostic Observation Schedule (Lord, Rutter, DiLavore, \& Risi, 1999), was administered by a pediatric neurologist specializing in autism spectrum conditions. Scores obtained also fell above the autism cut-off. An attempt to obtain a structural MRI of the brain was unsuccessful due to T.M.'s inability to cooperate with the procedures. However, according to caregiver report, a clinical MRI examination at the age of 5 did not reveal any specific structural abnormalities.

\section{General functioning}

T.M.'s low level of function in everyday life contrasted with some elements of his psychometric profile. T.M. made almost no eye contact, attended more to objects in his environment than to people, to which and to whom he tended to respond by sniffing. His autistic stereotypies ('stimming' behaviors) were frequent. T.M. manifested a high level of 
impulsive and obsessive behaviors as well as an inability to suppress aggressive behavior, typical of low-functioning individuals with autism. He often would behave in ways that showed an absence of perceived danger, e.g., running out into traffic, and could not be left alone for any length of time without the risk of endangering himself.

\section{Psychometric profile}

T.M.'s performance on psychometric tests reveals a theme of intact or superior performance on tasks demanding processing of auditory information, combined with impaired performance on tasks involving visual input to complex processing. T.M.'s performance on the Wechsler Intelligence Scale for Children, Third Edition (WISC-III; (Wechsler, 1991)) when he was 12 years 11 months of age demonstrated a Verbal IQ in the Superior range (VIQ=126; 96th percentile) but a Performance IQ in the Borderline range (PIQ=79; 8th percentile; Table 1). This is a discrepancy found in less than $5 \%$ of the standardization sample. Since T.M. has significant difficulties with oral output, he pointed to letters or numbers on a letter board to answer most of the questions of the verbal subtests, though in some cases he did respond orally. Although this procedure does not match that used in the standardization sample, the examiner (author N.A., an experienced clinical neuropsychologist) felt that the scores obtained were a good estimate of T.M.'s level of functioning at the time of the testing.

Visual confrontation naming in T.M. was evaluated using items from the Boston Naming Test in order to examine how T.M. was able to complete a verbal task using visual stimuli. T.M.'s severe articulation difficulty, combined with his need to circumlocute before naming an item, made timed oral responding impractical. The test items were therefore administered in a modified format in which T.M. wrote his responses rather than speaking them, and no time limit was used. Because of T.M.'s demonstrated strong vocabulary and because of a concern that he would have difficulty maintaining focus during a lengthy task, the test was restricted to the more complex items 30 through 60 . Including credit for the 30 basic items, which were not tested, T.M.'s total score on this modified Boston Naming Test was 53 (of 60), within the normal adult range. Three of T.M.'s seven failures were plausible interpretations of line drawings of objects with which he was unfamiliar ("dice" for dominoes, "harness" for muzzle, "maybe a fence" for trellis), and one was a plausible 
synonym ("bolt" for latch). Of the remaining incorrect responses, one was an item functionally related to the correct item ("door bell" for knocker), one was a physical description ("rope with a loop" for noose), and one was a contextual description ("old manuscripts and letters were in this format" for scroll). T.M.'s correct responses, rather than comprising single words, consisted of elaborate circumlocutions that included (and usually ended with) the correct response - e.g. "horned animal which is not a buffalo is a rhino", "a pod must be liked by squirrels it should be acorn." When asked why he wrote out these elaborate descriptions, and whether it would be possible for him to respond with just a single word, T.M. wrote, "I don't get the words so I try to describe it so I get it." Despite this difficulty with visual confrontation naming of objects, T.M. was able to repeat names that were spoken to him, to choose the correct name when presented with a list of possibilities, and to immediately name actions demonstrated to him. He was also able immediately to name objects from spoken descriptions (pencil, house, flute, scissors, tweezers).

T.M.'s scores on selected subsets of the Clinical Evaluation of Language Fundamentals Revised (Semel, Wiig, \& Secord, 1987) fell in the average to superior range (Listening to Paragraphs: 37th percentile; Semantic Relationships and Word Classes: 75th percentile; Oral Directions: 99th percentile). This cognitive profile of weak nonverbal skills and strong verbal skills is contrary to earlier studies of higher functioning individuals with autism (Lincoln, Allen, \& Kilman, 1995), although recent studies suggest that the V > NV profile is as common as the $\mathrm{NV}>\mathrm{V}$ profile in older children with autism (Joseph, Tager-Flusberg, \& Lord, 2002). Regardless of this controversy, what makes T.M. unique in the IQ sense is the above average verbal IQ without spoken language (see (Gernsbacher, 2004) for another such case).

To further explore T.M's visual and verbal processing, we conducted three more tests involving visual and verbal memory. T.M.'s performance on the Beery-Buktenica Developmental Test of Visual-Motor Integration (Beery, 1997), in which he had to copy drawings of meaningless complex geometric forms, was below expectations for age (standard score $=76$; 5th percentile). Though T.M. began with a somewhat unusual approach to copying the Rey-Osterrieth Complex Figure (i.e., copying the details on the 
right side of the page), he used a fairly mature, integrated approach when copying the figure's main elements (Akshoomoff \& Stiles, 1995). Despite his success in copying the figure, his free recall was poor. He drew a series of three boxes with an " $\mathrm{X}$ " in the middle, then wrote, "Nothing remember nothing". In contrast, T.M.'s cued recall of visual information in the NEPSY Memory for Faces test (Korkman, Kirk, \& Kemp, 1998) was in the average range (50th percentile), with correct identification of 12 of 16 faces in the immediate forced choice recognition condition. Moreover, TM showed a remarkable increase in performance (15 of 16 faces) after a 20-minute delay. Cued recall is generally stronger than free recall in autism as we found here (see review in (Ben Shalom, 2003)). However, the remarkable improvement of the delayed recall in T.M. is inconsistent with a recent study of high-functioning autism (D. L. Williams, Goldstein, \& Minshew, 2005). On the Wisconsin Card Sorting Test (Heaton, 1980), T.M. sorted all six categories within 74 cards and demonstrated no significant perseverative tendencies. Although some studies have demonstrated the individuals with autism perform poorly on this task (Ozonoff, 1995), results are not consistent (Minshew, Meyer, \& Goldstein, 2002).

\section{Vision and Audition}

T.M had normal (20/20) visual acuity in optometric testing, normal color vision (standard Ishihara color test), normal stereo vision, and normal hearing in standard audiometric testing. Additional psychophysical experiments with T.M. have shown superiority in perceptual tasks that tap local processing including auditory localization, visual search, and visual contrast discrimination, and impairment in global processing tasks of symmetry perception as well as contour integration: in a contour-in-noise detection test (Kovacs, Kozma, Feher, \& Benedek, 1999), T.M. performed worse than the norm for ages 5 to 6 (Bonneh and Pei, unpublished data). In addition, preliminary work indicated cross-modal interference between simultaneous visual, auditory, and tactile stimuli (Bonneh, Pei, Iversen, \& Merzenich, 2003).

\section{Communication}

T.M. was essentially non-verbal. His attempts at both single words and connected speech were only barely intelligible even if the context were known. He could not initiate communication on his own and was not able to interact with people unless his mother gave him the means to do so, such as setting up his laptop computer in close proximity and 
prompting him to begin typing. However, once prompted he was able to communicate independently without any physical facilitation, using a keyboard or a letter board or by handwriting. He was also able to answer questions related to his perception during the experiments. Vocal prompting by his mother using brief words such as "go" or "come on," sometimes repeated several times, was often necessary to re-establish attention and to enable response. This prompting did not cue a particular response, but rather appeared critical for initiating any response at all. For example T.M. would not move and point during the first few experimental trials unless prompted vocally by his mother. This initiation problem and the need for prompting usually diminished when the experiment reached a steady pace. Interestingly, when the sequence of trials was interrupted T.M. often failed to suppress his ongoing pointing. T.M. was able to sustain a question-and-answer period for extensive interval, sometimes 2 to 3 hours long, with 5-to-10-minute breaks every 10 to 15 minutes.

\section{Methods}

General experimental procedures. All experiments were done in the dark or in dim light. Stimuli were presented on a 17" CRT monitor running at $85 \mathrm{hz}$ refresh rate and located 70 $\mathrm{cm}$ from the subject, with two loudspeakers located at the two sides of the monitor. Tactile stimuli were generated via a sinusoidal signal delivered from the sound card to a custom amplifier developed by one of the authors (CIM) that drove a piezoelectric element without producing a noticeable sound. Stimuli were controlled by a dedicated program developed by the first author (YSB) and used in his previous studies. T.M. responded by pointing within a list of choices positioned so as to avoid spatial confounds, e.g. "left" and "right" positioned on an up-down axis. Although T.M. had some trouble maintaining fixation, this tended to be due to head or trunk movements and not just eye movements, and the experimenter verified that T.M. was looking at the monitor at the start of each trial. The experiments spanned four sets of 2 to 5 sessions over a period of one year, each session lasting for 2 to 3 hours, with 5-to-10-minute breaks every 10 to 15 minutes. In all experiments, ceiling performance meant $100 \%$ correct.

Control subjects. Eight normal children under 10 years of age (average of 8.5 years) serving as lower-bound controls for T.M.'s mental age, as determined by his PIQ (9 years 8 months), were tested to verify that normal subjects could easily perform the tasks. 
Experiment 1: Simple cross-modal extinction ("When I hear, my vision shuts down”).

The first experiment tested T.M.'s claims of cross-modal interference ("when I hear, my vision shuts down”), at the level of detection of salient stimuli. The test was a cross-modal version of a typical “extinction” test used in unilateral spatial neglect (e.g. (Driver \& Vuilleumier, 2001)), in which a stimulus on the left is extinguished from perception when presented simultaneously with another stimulus on the right. Here we simply replaced sides with perceptual modalities.

\section{Methods}

The paradigm is illustrated in Figure 1A. Simple visual, auditory, and tactile stimuli were presented. The visual stimulus was a high-luminance $\left(100 \mathrm{~cd} / \mathrm{m}^{2}\right)$ yellow square subtending $1.3^{\circ}$ of visual angle, flashed at fixation for $100 \mathrm{~ms}$. The auditory stimulus was a $100 \mathrm{~ms}$ burst of band-limited $(0-3 \mathrm{kHz})$ noise. The tactile stimulus was a 300 ms vibration of 250 hz. In each experiment, a pair of modalities (auditory-visual, visual-tactile, auditory-tactile) was tested. The task was to identify the modality or modalities of the stimulus, e.g. "visual", "auditory" or "both". The experiment was run in six sessions across 5 days and there were overall 60 trials each of visual, auditory, and visual-auditory stimuli, and 30 trials each of visual-tactile and auditory-tactile stimuli. All stimuli were set to be highly above detection threshold in isolation.

\section{Results}

Results are detailed in Figure 2. For each unimodal condition, performance was at or near ceiling. However, with simultaneous presentations of stimuli in two modalities, T.M. often reported only one. In these cases, the erroneous reports were of only one precedence: auditory stimuli were reported in the auditory-visual presentations (2a) and auditory-tactile presentations (2b), and visual stimuli were reported in the visual-tactile presentations (2c). Performance on the auditory-visual extinction paradigm (2a) was significantly lower than the unimodal visual response $(\mathrm{t}(10)=2.7, \mathrm{p}<0.02)$, demonstrating an explicit loss of awareness to a visual flash in the presence of a loud sound. All 8 of the mental-age matched controls performed at or near ceiling (around 100\%) on the audio-visual extinction test. 


\section{Discussion}

The results confirmed the claims that T.M. is affected by severe cross-modal interference, which occurs even at the level of detection of highly salient stimuli without any apparent attentional load. Interference under such conditions does not occur in normal observers, as shown by the 8 children of the control group as well as a previous study which found no penalty in dividing attention between vision and audition in simple non-speeded detection tasks even at threshold (A. M. Bonnel \& Hafter, 1998). However, interference does occur in normal observers when a speeded and time-restricted response is required. In this case naïve observers typically fail to respond to supra-threshold tones when presented simultaneously with a visual flash, a finding known as the Colavita effect (Colavita, 1974; Sinnett, Spence, \& Soto-Faraco, 2007) which demonstrates a visual bias in selective attention. This suggests that T.M.'s extinction effect could be a highly exaggerated form of a bias found also in normal observers, but one that occurs without a time restriction or an external load and in a different modality (auditory rather than visual dominance). As such, it appears similar in nature to the pathological extinction across space found in hemi-neglect patients (Driver \& Vuilleumier, 2001). Note that the results do not suggest the total inability to see and hear at the same time ("when I hear my vision shuts down"), but only the occasional extinction of transient salient stimuli by other transient stimuli from another modality. The intermittent nature of the effect is indicated by the observed variability (but not improvement or learning) of the audio-visual extinction results across days as well as large variability in tactile sensitivity in T.M. This instability could be common in autism, as we have recently studied another autism-spectrum case reporting large fluctuations in perceptual salience and load, with intermittent perceptual collapses (Bonneh, Popple, Howitt, \& Adini, 2007)). The possibility that T.M. has difficulty reporting any two perceptual events is explored and rejected in the following experiments.

Experiment 2: Cross-modal extinction in space \& time ("I need time to prepare my eyes").

In this experiment we further explored the auditory-visual interference effect found in experiment 1 , replacing the task from reporting modalities to reporting relative position while introducing a time lag between the stimuli. 


\section{Method}

The stimulus (Figure 1B) consisted of the auditory and visual elements described in Experiment 1, presented at lateral locations and separated by various stimulus onset asynchronies (SOA's). The auditory stimulus was presented from one of the two loudspeakers at $25^{\circ}$ of eccentricity, and the visual stimulus was presented $15^{\circ}$ left or right of fixation. In each case, T.M. was asked to tell whether the auditory and visual stimuli had been presented on the same or opposite sides. Each block comprised 32 trials (8 per spatial combination), and used a constant SOA. The auditory stimulus was always presented first since preliminary testing with T.M. showed that this condition was more difficult. The SOA's varied from $2.5 \mathrm{~s}$ (easy) to $0.5 \mathrm{~s}$ (difficult) in steps of $0.5 \mathrm{~s}$, and also included an additional SOA of $0.3 \mathrm{~s}$. Each block was repeated 3 to 4 times on four consecutive days and on one additional day after 3 months. To verify that T.M. was able to process positional information from each modality in isolation, the experiment was first done within modalities, i.e. by presenting two sounds or two patches on the same or different sides.

\section{Results}

Results are shown in Figure 3. Percentage correct is plotted as a function of the temporal interval between the onsets of the sound and the flash. In agreement with experiment 1 , T.M. had no difficulty reporting relative position within modality (visual-visual and auditory-auditory), where he was able to perform at ceiling at SOA's as low as $0.5 \mathrm{~s}$. In contrast, T.M. had an initial difficulty performing the cross-modal (auditory-visual) task at SOA's less than $1.5 \mathrm{~s}$, though performance was better at $2.5 \mathrm{~s}$ and $3 \mathrm{~s}$ (Figure 3a, "days 1-2" curve). When asked to explain his performance he said, "I need time to prepare my eyes" (or ears) and noted that a rapid switch between modalities was "painful" for him. The average results for the first two days were $85 \%$ correct for $1.5 \mathrm{~s}$, but close to chance performance for the $1 \mathrm{~s}$ SOA $(t(4)=4.4, \mathrm{p}<0.047)$. The data also reveal a marked improvement during the four days of the initial testing period, with average performance on days 3 and 4 at $90 \%$ for the $1 \mathrm{~s}$ SOA although still almost at chance level for $0.5 \mathrm{~s}(t(4)=5.6$, $\mathrm{p}<0.02$ for SOA of 1 and $0.5 \mathrm{~s}$ ). Interestingly, the performance after three months (day 5) regressed to baseline. In Figure 3b the similar audio-visual data of days 1-2 and 5 are replotted separately for the same and different conditions. The results show that T.M. made significantly more errors in the 'different' conditions, i.e. he reported audio-visual stimuli 
coming from different sides as coming from the same side. The mental-age matched controls performed the task in all conditions without difficulty, at or near ceiling (data shown in Figure 3 only for the audio-visual stimuli).

\section{Discussion}

This experiment further demonstrated that T.M. could easily process and report two items presented within a single modality but had difficulty in processing simple salient stimuli presented close together in time in different modalities. Under such conditions, information coming from the weaker modality, such as positional information, could be extinguished, e.g via "capture" of the visual location by the sound location.

\section{Experiment 3: Cross-modal Stroop-like effect ("I cannot ignore the word I hear..., I} am confused").

This experiment tested cross-modal interference at the level of language processing. We applied a cross-modal Stroop paradigm previously tested with children (Hanauer \& Brooks, 2003), modified for response by pointing rather than speaking, and measuring accuracy rather than reaction time.

\section{Method}

The paradigm is illustrated in Figure 1C. T.M. was asked to identify the visual stimulus while ignoring the simultaneously presented auditory stimulus. Visual stimuli were highluminance red or blue patches, subtending $2^{\circ}$, presented for $100 \mathrm{~ms}$ on a black background. Auditory stimuli were loud recordings of the words "red" and "blue", the irrelevant words "cat" and "dog", band-limited noise, and silence. T.M. responded by pointing to a drawing of a red or a blue patch, in one response condition, or by pointing to the written word "red" or "blue", in the other condition. In a modification of this experiment, the colored patches were replaced by large yellow "+" and "-" symbols, and the spoken words "red" and "blue" by the spoken words "plus" and "minus". In another condition, the symbols "+" and "-" were presented in red and blue respectively (fixed color-symbol assignment) and spoken color words were used as distractors. Each experimental block comprised 32 randomly ordered trials -8 for each of the 4 sound conditions - and each block was repeated 4 to 5 times. 


\section{Results}

The results for color identification appear in Figure 4a. In the case of irrelevant sounds (silence, noise, or irrelevant words), as well as valid spoken words (e.g. "blue" for a blue patch), performance was near ceiling. However, for invalid words T.M. tended to respond according to the spoken word $(t(6)=21.4, \mathrm{p}<0.00001$ for invalid different from valid). In some cases T.M. reported explicit perceptual misclassifications (e.g. "I saw it blue" when the patch was red and the word was "blue"). When an SOA of $1 \mathrm{~s}$ was introduced between the patch and the sound, performance for invalid sounds increased to $50 \%$ but still differed significantly from that for valid words $(t(4)=7.1, \mathrm{p}<0.045)$. Interestingly, the type of response cue made a difference: more errors occurred when T.M. was pointing to text (almost 0\% correct) than when he was pointing to drawings (20\% correct). The results for the symbol identification task (words "plus" or "minus" and the corresponding symbols) were similar and are presented in Figure 4b. The mental-age matched controls performed at or very near ceiling.

\section{Discussion}

This experiment shows that T.M. is stimulus-bound to spoken words when attempting to report simultaneous, incongruent visual stimuli. Under these conditions, the stimuli coming from the auditory channel do not merely slow the response as in the classical Stroop effect, but take over completely, making this result extremely abnormal.

\section{Experiment 4: Cross-channel color-form interference ("These stimuli are equally powerful; I get one and can only guess the other")}

This experiment tested interference or competition within vision. We compared the ability to process color and form simultaneously with the processing of two forms and with the processing of motion and form.

\section{Method}

The paradigm is illustrated in Figure 1D. A high-luminance color patch (red or blue, $2^{\circ}$ in size) was presented for 100 ms close to fixation on a black background below a yellow "+" or "-" symbol of similar size. T.M. was asked to report the color and then the symbol or, in 
another reporting condition, the symbol and then the color. In a control task, a second "+" or "-" symbol was substituted for the colored patch and the task was to report the two symbols. Two modifications of this experiment were used. In the first single-object condition, the color and form attributes of interest were combined in a single, colored "+" or "-" symbol and the task was to report the color and the symbol. In the second motion-form condition, the color patch was presented in lateral motion (2\%) along the horizontal axis for $250 \mathrm{~ms}$ and the task was to report the direction of motion as well as to identify the symbol. Eight trials per color-symbol combination were presented in random order, and each condition was tested 3 to 5 times. The color-symbol experiment was repeated for two temporal separations (300 ms and $1 \mathrm{~s}$ ) between the color and the symbol.

\section{Results}

The results are shown in Figure 5. While T.M. could easily report two symbols (Figure 5a, rightward bar), performance for reporting both color and symbol approached chance for the second item reported. Figure $5 \mathrm{~b}$ shows that when the symbol and the color patch were separated in time, performance reached $100 \%$ correct at $1 \mathrm{~s}$ separation, with improved identification already at $300 \mathrm{~ms}$ separation. The results for the single-object condition in which form and color were combined in one symbol (e.g. a red plus) were similar to simultaneous presentation: 62\% correct (average across both tasks, SE=6\%). In contrast, performance for reporting both motion direction and symbol in the motion-form condition was $87 \%$ correct (average across both tasks, SE=3.6\%). The mental-age matched controls $(\mathrm{N}=8)$ performed the basic color and symbol task at or very near ceiling.

\section{Discussion}

This experiment established that two simultaneous stimuli could be reported within a single perceptual channel (two forms), but not in different channels (color and form). The deficit persisted even when the two stimuli were combined in one object but disappeared when a temporal separation was introduced, similar to the result of experiment 2 between sound and vision. Interestingly, no deficit was found when the color task was replaced by a motion laterality judgment task, suggesting that motion and form do not compete significantly, or that the attentional demand for determining the motion direction was lower than that for color identification. 


\section{Experiment 5: Unimodal shifting of visual spatial attention.}

One critical component in dealing with multiple and competing stimuli as investigated in the previous experiments is the ability to disengage attention from one object and shift it to another. This experiment tested T.M.’s visual spatial attention shifting.

\section{Methods}

The paradigm for visual spatial attention shifting was a modified version of one applied in previous studies of adults with high-functioning autism (Belmonte, 2000; Belmonte \& Yurgelun-Todd, 2003). Red or green squares subtending $1.8^{\circ}$ and centered $3.0^{\circ}$ lateral and $5.1^{\circ}$ superior to a fixation cross were flashed at $9 \mathrm{hz}$ (55 ms on, $55 \mathrm{~ms}$ off) in left and right hemifields. At any given time, one side of the display was attended and the other was ignored; ratios of targets to non-targets were identical in both hemifields. On detecting a target (red) stimulus at the attended location, T.M. had to shift his attention to the opposite side, and to indicate the direction of the shift by pressing a button on the left or right side of a response box. These responses were scored as correct if they occurred between $0.2 \mathrm{~s}$ and 6 s following an attended target in the hemifield contralateral to the direction of the response. A total of 111 attended targets was presented in 14 blocks of $60 \mathrm{~s}$ each. On the basis of modal peaks in pilot data, results were classified into three bins based on the length of the shift interval, that is, the amount of time elapsed between the current target and the most recent correctly identified target: less than $2.5 \mathrm{~s}, 2.5 \mathrm{~s}$ to $6 \mathrm{~s}$, and $6 \mathrm{~s}$ or longer.

\section{Results}

For shift intervals less than 2.5 s, T.M. correctly identified 10 of 18 targets (55.6\%). For intervals between $2.5 \mathrm{~s}$ and 6 s, T.M. correctly identified 19 of 23 targets (82.6\%). For intervals greater than 6s, T.M. correctly identified 20 of 54 targets (37.0\%). Fisher's exact test revealed a significant difference between these accuracy rates for the $>6 \mathrm{~s}$ bin versus the 2.5-6s bin (two-sided $\mathrm{p}=0.0003679$ ) and a trend for the $0-2.5 \mathrm{~s}$ bin versus the 2.5-6s bin (two-sided $\mathrm{p}=0.0869$ ). The average response latency was $2.426 \mathrm{~s}$ (SD $1.229 \mathrm{~s}$ ).

\section{Discussion}

This experiment replicated in T.M. the finding of slowed shifting of attention, which has been established in the case of high-functioning autism (T.M.'s impairment at shifts spaced 
more closely than 2.5 s), and also, consistent with our clinical impression of T.M., indicated a deficit in sustaining attention (impairment at shifts spaced farther apart than $6 \mathrm{~s}$ ). This latter deficit is not generally present in high-functioning autism (Goldstein, Johnson, \& Minshew, 2001) but may be more common in low-functioning cases.

\section{General Discussion}

We have described a case of a child with autism in which two extreme and possibly related aspects of autism were revealed: (1) a discrepancy between high verbal and low visuospatial abilities measured by psychometric testing, which was combined with a lack of spoken language and many other symptoms typically found in low-functioning individuals with autism, and (2) a severe difficulty in processing stimuli from different modalities or perceptual channels close together in time, with a preference for processing auditory stimuli over visual and tactile stimuli. In a series of psychophysical experiments, we investigated cross-modal interference at different levels of processing and found that abnormal processing of multimodal stimuli occurred without any apparent attentional load and with highly salient stimuli, thus providing the first empirical evidence for mono-channel perception in autism, a phenomenon that has been described only anecdotally until now.

In the following sections we analyze and interpret the results at different theoretical levels. We consider a simple sensory masking explanation, discuss the ideas underlying "monotropism" and mono-channel perception (Murray et al., 2005), and follow up with a discussion of a general winner-takes-all principle as underlying much of the autistic behavior that we measured. Finally we suggest a possible explanation for T.M.'s high verbal IQ and discuss the implications of the case of T.M. for the study of autism in general.

\section{Cross-modal extinction as an effect of sensory masking}

According to a "sensory" explanation, large differences in sensory gains across different channels (hyper- and hypo-sensitivity) could produce sensory masking, in which enhanced input in one modality masks weaker input from other channels. The evidence for hyper- and hypo-sensitivity (Iarocci \& McDonald, 2006; O'Neill \& Jones, 1997) and enhanced perceptual discrimination (Bertone, Mottron, Jelenic, \& Faubert, 2005; A. Bonnel et al., 
2003; Mottron, Dawson, Soulieres, Hubert, \& Burack, 2006) in autism supports such an interpretation, and T.M. himself often exhibited hyposensitivity to parts of his body. However, enhanced perceptual input by itself is unlikely to account for the current results, because T.M.'s sensory sensitivity appeared normal for both vision (contrast sensitivity, acuity) and audition (audiometric test), and because the tasks and stimuli were largely insensitive to variations in stimulus strength (stimuli were salient and the tasks did not require fine discriminations). Nevertheless, the idea of a high sensory or perceptual gain is consistent with T.M.'s description of sensory and perceptual overload. By his own assessment, T.M. resolves his perceptual environment by focusing on one sensory modality at a time, because "trying to use all the senses turns into total chaos.” This observation of specifically multi-sensory impairment leads to "attentional" explanations discussed below.

\section{Monotropism, mono-channel perception, and cross-modal extinction}

Murray and colleagues (Murray et al., 2005) have recently proposed that an abnormally narrow distribution of attention or "monotropism" is central to the autistic condition and accounts for a multitude of autistic symptoms, including perceptual abnormalities and restricted interests. Our results are consistent with monotropism in the sense of monochannel processing, and specifically across sensory modalities and processing modules. Although T.M.'s perception, as we observed, is characterized by a widely tuned, sensorydriven style of processing in which he may be attracted to peripheral stimuli at any time, he often gets "stuck", focusing on a single channel, object or stimulus part, while ignoring the remaining perceptual information. Whereas the theory of monotropism emphasizes a competition for limited attentional resources, our results indicate mono-channel perception even without any apparent perceptual load, and in tasks whose resource demands are very limited, such as simultaneous detection of sound and light (Experiment 1). This discrepancy could be resolved by positing that in people with autism even a normal level of background stimuli creates an ongoing perceptual load (e.g. due to inherently enhanced perception (Mottron et al., 2006)), or alternatively, by suggesting that in autism mono-channel perception occurs automatically as a fundamental property of the perceptual system perhaps one developed and engrained as a compensatory cognitive strategy in response to a world of intractably multi-modal stimuli. This aspect of T.M.'s perceptual system, which we refer to as a winner-takes-all mode, is discussed next. 
Cross-modal extinction as a manifestation of winner-takes-all processing in autism

A general pattern of behavior emerged in all the experiments: a winner-takes-all mode of processing in which weak stimuli or representations are extinguished by more salient ones. This effect was found at the levels of detection, where audition extinguished visual and tactile stimuli (Experiment 1); spatial position encoding, where simultaneous or near-simultaneous stimuli were erroneously co-located (Experiment 2); stimulus-response mapping, where the irrelevant word eliminated the relevant response (Experiment 3); color and form representation, where prior presentation of one stimulus interfered with perception of the other (Experiment 4); and visual spatial attention, where a prior focus within one hemifield interfered abnormally strongly with the perception of later stimuli in the other (Experiment 5). This combination of results reveals the properties of this winner-takes-all effect: it does not depend on the presence of an external 'attentional load,' as shown in Experiment 1 using just two simple and highly salient stimuli. It is general across tasks and stimuli and is not subject to cognitive control, since it occurred when T.M. had to attend to stimuli (Experiments 1, 2, 4, and 5) as well as when he had to ignore stimuli (Experiment 3). This type of winner-takes-all processing in which a stronger representation dominates behavior while extinguishing a weaker representation may be a general property of autism, possibly related to observations of impaired contextual processing (Frith, 1989; Happe, 1996), stimulus over-selectivity (Lovaas et al., 1979) and impaired episodic memory with extinction of contextual details ((Ben Shalom, 2003).

A related "attentional" interpretation is suggested by Experiment 5's finding of deficits in shifting and sustaining spatial attention. A slow attentional disengagement (Posner, Walker, Friedrich, \& Rafal, 1987) could explain T.M.'s difficulty in processing multiple stimuli occurring close together in time ("I need time to prepare my eyes"). Computationally, winner-takes-all processing and slow attentional disengagement both could be explained in terms of reduced inhibition, either locally or centrally (Fukai \& Tanaka, 1997; Koch \& Ullman, 1985). In the absence of local inhibition, the normal process of inhibiting the selected "winner" and automatically shifting to the next most salient location or module may be slowed, consequently preventing the rapid deployment of attention to competing stimuli. Such an interpretation is consistent with the evidence for reduced inhibition in autism (Rubenstein \& Merzenich, 2003). In addition to this winner- 
takes-all deficit in voluntary shifting of attention away from a prior salient focus, T.M.'s deficit in sustained attention may be explained by winner-takes-all attentional capture by occasional salient background stimuli. This winner-takes-all processing style thus is capable of explaining apparently complementary deficits in shifting and sustaining. Difficulty shifting attention between auditory and visual stimuli has previously been reported for individuals with autism (Courchesne et al., 1994). In that study, however, the deficit appeared to be due to difficulty shifting the focus of attention rapidly from one modality to the other rather than difficulty disengaging attention (as evidenced by no difficulty with false alarm errors).

What could be the cortical site or sites for the observed cross-modal extinction or winner-takes-all processing? Accumulating evidence suggests that areas in the parietal lobe are engaged in multimodal processing and their damage underlies the non-spatial extinction found in neglect patients (Husain \& Rorden, 2003). A more concrete candidate is the superior temporal sulcus (STS), known to be involved in polysensory processing, such as in audio-visual association during speech perception (Macaluso, George, Dolan, Spence, \& Driver, 2004; Wright, Pelphrey, Allison, McKeown, \& McCarthy, 2003) and cross-modal attention shifting (Macaluso, Frith, \& Driver, 2001). There is some direct evidence for abnormal operation of STS in autism (Boddaert et al., 2004; Pelphrey, Morris, \& McCarthy, 2005; Waiter et al., 2004), and evidence for abnormal spatial extinction of competing stimuli following a lesion to STS in monkeys (Luh, Butter, \& Buchtel, 1986). Nevertheless, the evidence for STS abnormality in autism and appreciable STS function in general is not specific enough to determine whether it underlies the current results, which relate to crossmodal competition rather than integration or association.

It is interesting to speculate as to how T.M.'s naming difficulty (see Case Details) and his attention impairment discussed above may share computational structure. As the retrieval of a name is facilitated by the activation of semantically related terms which may help to exclude irrelevant responses (Mahon, Costa, Peterson, Vargas, \& Caramazza, 2007) a high degree of non-selective activation extending to unrelated terms might impair response. T.M.'s evocative circumlocutions may figure as an adaptive strategy, a sort of self-priming in which production of semantically related terms aims at seeding activity in the 
relevant region of the semantic network - recall his self-report "I don't get the words so I try to describe it so I get it." Such a mechanism may also contribute to T.M.'s seeming superiority at cued recall (Memory for Faces) as compared to free recall (Rey-Osterrieth Complex Figure).

High verbal IQ as the developmental product of an auditory-oriented cognitive style

What could explain T.M.'s paradoxically high verbal intelligence? T.M.'s preference for processing auditory stimuli over visual and tactile stimuli could have supported verbal skill development via a fixation on words and language. However, given the general nature of his verbal intelligence and his severe abnormalities and seemingly low level of function, this might not be the full explanation. A further element could be his unique developmental history of extensive human prompting. This rapid auditory prompting appeared critical in initiating a response during the experiments (see "Case details”), and was often necessary in order for T.M. to initiate and to follow even a simple sequence of actions such as moving his gaze to three people in the room, one after the other, an exercise which he performed slowly and with great effort. In contrast, during self-stimulatory and stereotyped movements, T.M. moved rapidly and fluently. T.M.'s difficulty in initiating actions could be related to his deficit in shifting attention in space or disengaging attention from a strong stimulus, and a product of the winner-takes-all mode of processing. We speculate that the rapid auditory prompting modulates arousal in T.M. in a manner similar to phasic alerting in stroke patients (Robertson, Mattingley, Rorden, \& Driver, 1998), increasing temporarily the total attentional capacity and preventing extinction.

What could explain T.M.'s lack of intelligible speech despite his high verbal intelligence? One explanation is a general motor deficit (apraxia) that prevents him from producing voluntary speech. This would be consistent with T.M.'s atypical poor performance on the block design test as well as his general clumsiness and apparently poor motor control in voluntary (but not involuntary) movements. An alternative explanation, which is related to his severe perceptual abnormalities investigated in the current study is indicated by T.M.'s claim that he does not hear himself when he speaks intentionally, a claim also reported to us recently by two other autistics. This suggests a mono-channel type of processing in which speech production extinguishes auditory perception of speech. In other words, T.M.'s 
attempts to produce speech extinguished his reception at that time and prevented normal processing of auditory feedback, which is critical for speech development (Borden, Harris, \& Raphael, 1994). Accordingly, if T.M. can only pay attention to or process one sensory or motor channel at once, he'll not be able to associate motor commands with their sensory consequences and will not know how to move his articulators to achieve a desired sound. The winner-takes-all interpretation we have suggested to account for the experimental data can thus be used to explain different behavioral abnormalities in T.M. It points to a potentially common and important abnormal computational pattern that may apply to different degrees across time (e.g. see discussion of experiment 1) and across different individuals, some of which fail to develop speech.

On the generality and implications of the case of T.M.

The case of T.M., so remarkable for his combination of severely autistic behaviors with a high verbal intelligence that allows him to perform complex psychophysical experiments, may illuminate the nature of cognitive functioning in many other people with lowfunctioning autism who are less able to communicate and to comply with experimental procedures, and who therefore escape neuroscientific attention. T.M. can hear, see, or feel touch in isolation, but often fails to see or to feel touch when he hears a sound and fails to feel touch when he sees a flash. We suggest that this phenomenon of cross-modal extinction reflects a general computational pattern of "winner takes all" processing, and could arise from a combination of inherent abnormalities (slow attentional disengagement, hemineglect-like competition) with compensatory strategies developed to avoid over-stimulation or to accommodate a bottleneck in simultaneous processing. While this suggestion is based on a single case, and thus should be considered with caution, the pattern of results reported here may not be unique to T.M., and in fact may be common to many people with lowfunctioning autism. Greater insight into such cases and their underlying causes will aid in the design of more appropriate and efficient interventions, allowing more of these patients to communicate with the world around them. 
Acknowledgements: We thank Drs William Hirstein, Stefano Baldassi, Russ Hamer, David Blake and Tony Norcia for valuable consultation. We thank Dr. Dorit Ben Shalom for helpful comments. We thank the Smith-Kettlewell Eye Research Institute for hosting most of the testing. We acknowledge the contribution of the written evaluation of T.M. as severely autistic by Drs Lorna Wing, Judith Gold and Beate Hermelin. The study was supported by Cure Autism Now and by a grant from the National Institute for Psychobiology in Israel to the first author. Special thanks to our subject T.M. and his mother for their enormous effort and collaboration. 


\section{References}

Akshoomoff, N. A., \& Stiles, J. (1995). Developmental trends in visuospatial analysis and planning: I. Copying a complex figure. Neuropsychology, 9, 364-377.

Allen, G., \& Courchesne, E. (2001). Attention function and dysfunction in autism. Front Biosci, 6, D105-119.

Beery, K. E. (1997). The Beery-Buktenica Developmental Test of Visual-Motor Integration (VMI) Administration, Scoring, and Teaching Manual (4th Edition Revised ed.). Parsippany, N.J: Modern Curriculum Press.

Belmonte, M. K. (2000). Abnormal attention in autism shown by steady-state visual evoked potentials. Autism, 4(3), 269-285.

Belmonte, M. K., \& Yurgelun-Todd, D. A. (2003). Functional anatomy of impaired selective attention and compensatory processing in autism. Brain Res Cogn Brain Res, 17(3), 651-664.

Ben Shalom, D. (2003). Memory in autism: review and synthesis. Cortex, 39(4-5), 1129-1138.

Bertone, A., Mottron, L., Jelenic, P., \& Faubert, J. (2005). Enhanced and diminished visuo-spatial information processing in autism depends on stimulus complexity. Brain, 128(Pt 10), 2430-2441.

Boddaert, N., Chabane, N., Gervais, H., Good, C. D., Bourgeois, M., Plumet, M. H., et al. (2004). Superior temporal sulcus anatomical abnormalities in childhood autism: a voxel-based morphometry MRI study. Neuroimage, 23(1), 364-369.

Bonneh, Y. S., Pei, F., Iversen, P., \& Merzenich, M. M. (2003). Cross-modal extinction in autism: a case report. Perception, supplement (ECVP 2003).

Bonneh, Y. S., Popple, A., Howitt, D. H., \& Adini, Y. (2007). Why do they suddenly cover their ears? Investigation of a case of ASD who reports intermittent auditory visual and crossmodal hyper states. Paper presented at the International Meeting on Autism Research (IMFAR).

Bonnel, A., Mottron, L., Peretz, I., Trudel, M., Gallun, E., \& Bonnel, A. M. (2003). Enhanced pitch sensitivity in individuals with autism: a signal detection analysis. J Cogn Neurosci, 15(2), 226-235.

Bonnel, A. M., \& Hafter, E. R. (1998). Divided attention between simultaneous auditory and visual signals. Percept Psychophys, 60(2), 179-190.

Borden, G. J., Harris, K. S., \& Raphael, L. J. (1994). Speech science primer: physiology, acoustics, and perception of speech (3rd ed.). Baltimore: Williams \& Wilkins.

Braun, J., \& Julesz, B. (1998). Withdrawing attention at little or no cost: detection and discrimination tasks. Percept Psychophys, 60(1), 1-23.

Colavita, F. B. (1974). Human sensory dominance. Percept Psychophys, 16( ), 409-412.

Courchesne, E., Townsend, J., Akshoomoff, N. A., Saitoh, O., Yeung-Courchesne, R., Lincoln, A. J., et al. (1994). Impairment in shifting attention in autistic and cerebellar patients. Behav Neurosci, 108(5), 848-865.

Driver, J., \& Vuilleumier, P. (2001). Perceptual awareness and its loss in unilateral neglect and extinction. Cognition, 79(1-2), 39-88.

Frith, U. (1989). Autism: explaining the enigma. Oxford: Basil Blackwell.

Fukai, T., \& Tanaka, S. (1997). A simple neural network exhibiting selective activation of neuronal ensembles: from winner-take-all to winners-share-all. Neural Comput, 9(1), 77-97.

Gernsbacher, M. (2004). Language is More than Speech: A Case Study. Journal of Developmental and Learning Disorders,, 8, 81-98.

Goldstein, G., Johnson, C. R., \& Minshew, N. J. (2001). Attentional processes in autism. J Autism Dev Disord, 31(4), 433-440.

Grandin, T. (1995). Thinking in pictures and other reports from my life with autism. New York: Vintage Books.

Hanauer, J. B., \& Brooks, P. J. (2003). Developmental change in the cross-modal Stroop effect. Percept Psychophys, 65(3), 359-366.

Happe, F. G. (1996). Studying weak central coherence at low levels: children with autism do not succumb to visual illusions. A research note. J Child Psychol Psychiatry, 37(7), 873-877.

Heaton, R. K. (1980). A Manual for the Wisconsin Card Sorting Test. Odessa, FL: Psychological Assessment Resources, Inc.

Husain, M., \& Rorden, C. (2003). Non-spatially lateralized mechanisms in hemispatial neglect. Nat Rev Neurosci, 4(1), 26-36.

Iarocci, G., \& McDonald, J. (2006). Sensory Integration and the Perceptual Experience of Persons with Autism. J Autism Dev Disord, 1-14.

Joseph, R. M., Tager-Flusberg, H., \& Lord, C. (2002). Cognitive profiles and social-communicative 
functioning in children with autism spectrum disorder. J Child Psychol Psychiatry, 43(6), 807-821.

Kanner, L. (1943). Autistic disturbances of affective contact. Nervous Child, 2, 217-250.

Koch, C., \& Ullman, S. (1985). Shifts in selective visual attention: towards the underlying neural circuitry. Hum Neurobiol, 4(4), 219-227.

Korkman, M., Kirk, U., \& Kemp, S. (1998). NEPSY: A developmental neuropsychological assessment. San Antonio: Psychological Corporation.

Kovacs, I., Kozma, P., Feher, A., \& Benedek, G. (1999). Late maturation of visual spatial integration in humans. Proc Natl Acad Sci U S A, 96(21), 12204-12209.

Lawson, W. (2003). Life behind glass. London: Jessica Kingsley Publishers.

Lincoln, A. J., Allen, M. H., \& Kilman, A. (1995). The assessment and interpretation of intellectual abilities in people with autism. In E. Schopler \& G. B. Mesibov (Eds.), Learning and cognition in autism (pp. 89-117). New York: Plenum Press.

Lord, C., Rutter, M., DiLavore, P. C., \& Risi, S. (1999). Autism diagnostic observation schedule. Los Angeles: Western Psychological Services.

Lord, C., Rutter, M., \& Le Couteur, A. (1994). Autism Diagnostic Interview-Revised: a revised version of a diagnostic interview for caregivers of individuals with possible pervasive developmental disorders. $J$ Autism Dev Disord, 24(5), 659-685.

Lovaas, O. I., Koegel, R. L., \& Schreibman, L. (1979). Stimulus overselectivity in autism: a review of research. Psychol Bull, 86(6), 1236-1254.

Luh, K. E., Butter, C. M., \& Buchtel, H. A. (1986). Impairments in orienting to visual stimuli in monkeys following unilateral lesions of the superior sulcal polysensory cortex. Neuropsychologia, 24(4), 461470.

Macaluso, E., Frith, C. D., \& Driver, J. (2001). Multimodal mechanisms of attention related to rates of spatial shifting in vision and touch. Exp Brain Res, 137(3-4), 445-454.

Macaluso, E., George, N., Dolan, R., Spence, C., \& Driver, J. (2004). Spatial and temporal factors during processing of audiovisual speech: a PET study. Neuroimage, 21(2), 725-732.

Mahon, B. Z., Costa, A., Peterson, R., Vargas, K. A., \& Caramazza, A. (2007). Lexical selection is not by competition: a reinterpretation of semantic interference and facilitation effects in the picture-word interference paradigm. J Exp Psychol Learn Mem Cogn, 33(3), 503-535.

Minshew, N. J., Meyer, J., \& Goldstein, G. (2002). Abstract reasoning in autism: a dissociation between concept formation and concept identification. Neuropsychology, 16(3), 327-334.

Mottron, L., Dawson, M., Soulieres, I., Hubert, B., \& Burack, J. (2006). Enhanced perceptual functioning in autism: an update, and eight principles of autistic perception. J Autism Dev Disord, 36(1), 27-43.

Murray, D., Lesser, M., \& Lawson, W. (2005). Attention, monotropism and the diagnostic criteria for autism. Autism, 9(2), 139-156.

O'Neill, M., \& Jones, R. S. (1997). Sensory-perceptual abnormalities in autism: a case for more research? J Autism Dev Disord, 27(3), 283-293.

Ozonoff, S. (1995). Reliability and validity of the Wisconsin Card Sorting Test in studies of autism. Neuropsychology, 9, 491-500.

Pelphrey, K. A., Morris, J. P., \& McCarthy, G. (2005). Neural basis of eye gaze processing deficits in autism. Brain, 128(Pt 5), 1038-1048.

Posner, M. I., Walker, J. A., Friedrich, F. A., \& Rafal, R. D. (1987). How do the parietal lobes direct covert attention? Neuropsychologia, 25(1A), 135-145.

Robertson, I. H., Mattingley, J. B., Rorden, C., \& Driver, J. (1998). Phasic alerting of neglect patients overcomes their spatial deficit in visual awareness. Nature, 395(6698), 169-172.

Rubenstein, J. L., \& Merzenich, M. M. (2003). Model of autism: increased ratio of excitation/inhibition in key neural systems. Genes Brain Behav, 2(5), 255-267.

Semel, E., Wiig, E. H., \& Secord, W. (1987). Clinical Evaluation of Language Fundamentals (Revised ed.). New York: Psychological Corp.

Sinnett, S., Spence, C., \& Soto-Faraco, S. (2007). Visual dominance and attention: the Colavita effect revisited. Percept Psychophys, 69(5), 673-686.

Waiter, G. D., Williams, J. H., Murray, A. D., Gilchrist, A., Perrett, D. I., \& Whiten, A. (2004). A voxel-based investigation of brain structure in male adolescents with autistic spectrum disorder. Neuroimage, 22(2), 619-625.

Wechsler, D. (1991). Wechsler Intelligence Scale for Children (Third ed.). San Antonio: The Psychological Corporation.

Williams, D. (1996). Autism: an inside-out approach. London and Philadelphia: Jessica Kingsley Publishers.

Williams, D. L., Goldstein, G., \& Minshew, N. J. (2005). Impaired memory for faces and social scenes in 
autism: clinical implications of memory dysfunction. Arch Clin Neuropsychol, 20(1), 1-15.

Wright, T. M., Pelphrey, K. A., Allison, T., McKeown, M. J., \& McCarthy, G. (2003). Polysensory

interactions along lateral temporal regions evoked by audiovisual speech. Cereb Cortex, 13(10), 1034-1043. 
Table 1. WISC-III Results

\begin{tabular}{|c|c|c|c|c|c|}
\hline \multicolumn{3}{|c|}{$\underline{\text { Scaled }}$} & \multicolumn{3}{|c|}{$\underline{\text { Scaled }}$} \\
\hline$\underline{\text { Verbal Tests }}$ & $\underline{\text { Score }}$ & $\underline{\text { Percentile }}$ & $\underline{\text { Performance Tests }}$ & $\underline{\text { Score }}$ & $\underline{\text { Percentile }}$ \\
\hline Information & 18 & 99 & Picture Completion & 6 & 9 \\
\hline Similarities & 15 & 95 & Picture Arrangement & 3 & 1 \\
\hline Arithmetic & 9 & 37 & Block Design & 7 & 16 \\
\hline Vocabulary & 15 & 95 & (Symbol Search & 10 & 50) \\
\hline Comprehension & 14 & 91 & & & \\
\hline
\end{tabular}

Verbal IQ

$126\left(96^{\text {th }}\right.$ percentile)

Performance IQ $\quad 79 \quad\left(8^{\text {th }}\right.$ percentile $) \quad$ [prorated from 4 subtests]

Full Scale IQ $\quad 104 \quad\left(61^{\text {st }}\right.$ percentile $)$

[NOTE: Scaled scores have a mean of 10 with a standard deviation of 3. IQ scores have a mean of 100 with a standard deviation of 15.] 
Cross-modal Extinction in Space-Time

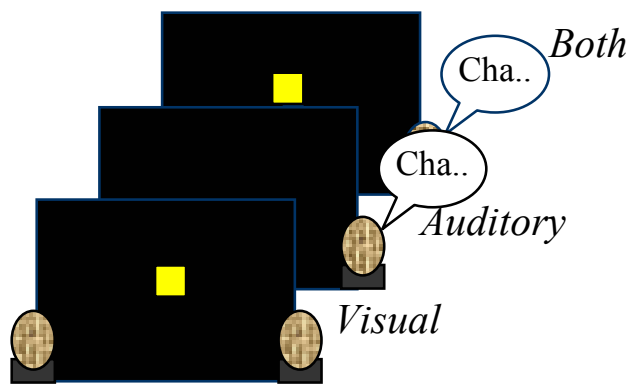

Did you hear, see, or both?

$\mathbf{C}$

Cross-modal "Stroop"

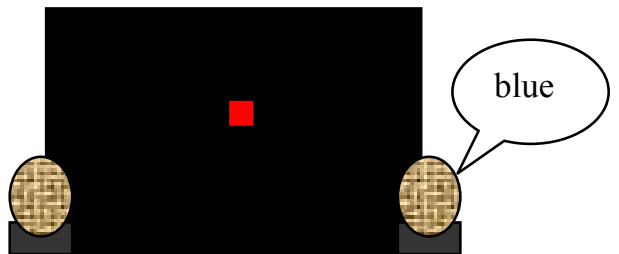

What was the color (ignore the sound)?

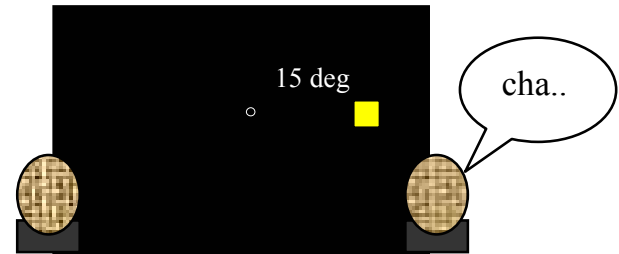

Sound Flash

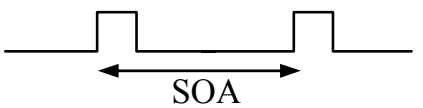

Same side or different sides?

Cross-module (color-form) suppression

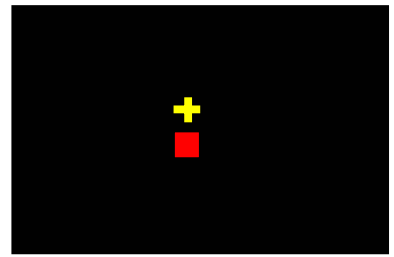

What was the color? What was the sign?

Figure 1 - The 4 experimental paradigms used to test cross-modal interference. (A) Illustrates the audio-visual cross-modal extinction test, where a bright yellow patch, a band noise, or both were randomly presented and the task was to respond with "visual", "auditory" or "both". A similar paradigm but with different stimuli was used to test auditory-tactile and visual-tactile extinction. (B) Illustrates the test for crossmodal extinction in space-time for the auditory-visual condition. The task was to judge whether two stimuli appeared on the same or different sides, with auditory stimuli (short band noise that sounds like "cha..") presented to one of the lateral speakers and visual stimuli (patches of light) presented to one side of the screen after a delay (SOA). (C) Illustrates the cross-modal "Stroop" effect. T.M. had to report the color of a briefly presented patch and ignore the spoken color word (e.g. "blue" for a red patch). (D) Illustrates the color-form interference effect. T.M. had to report both the yellow symbol (+/-) and the color of the patch (red/blue) that were briefly presented. 

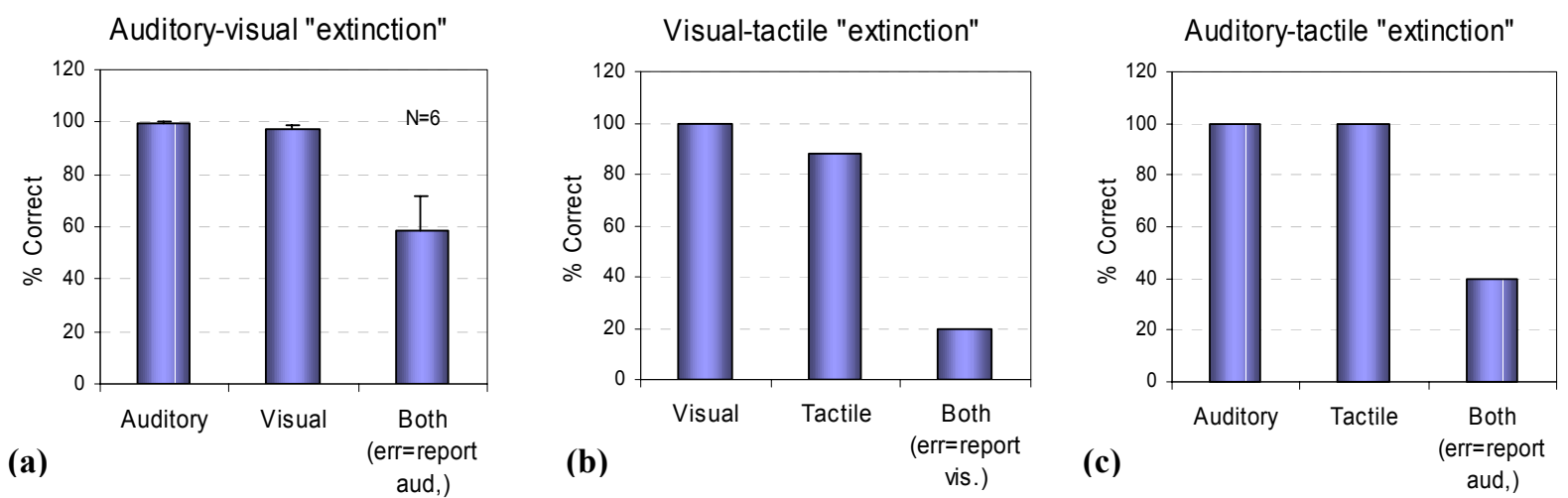

Figure 2 - Results for the cross-modal extinction experiment (1). Each graph shows the proportion of correct detections for unimodal and bimodal stimuli. Whereas errors in the bimodal cases (simultaneous stimuli from two modalities) could reflect misses of one or the other stimulus, in practice, the errors were all of one type: misses of visual stimuli in (a), and misses of tactile stimuli in (b) and (c). Each session included 30 trials of each type (two unimodal types and one bimodal). Error bars in (a) denote 1 standard error across 6 sessions collected in different days, with the main variability for bimodal stimuli occurring across days - a high detection of simultaneous auditory and visual stimuli during 2 of the days, and a low detection (14\%-38\%) in other days. The single session of each of the tactile experiments produced similar results, with a selective impairment with simultaneous bimodal stimuli. 


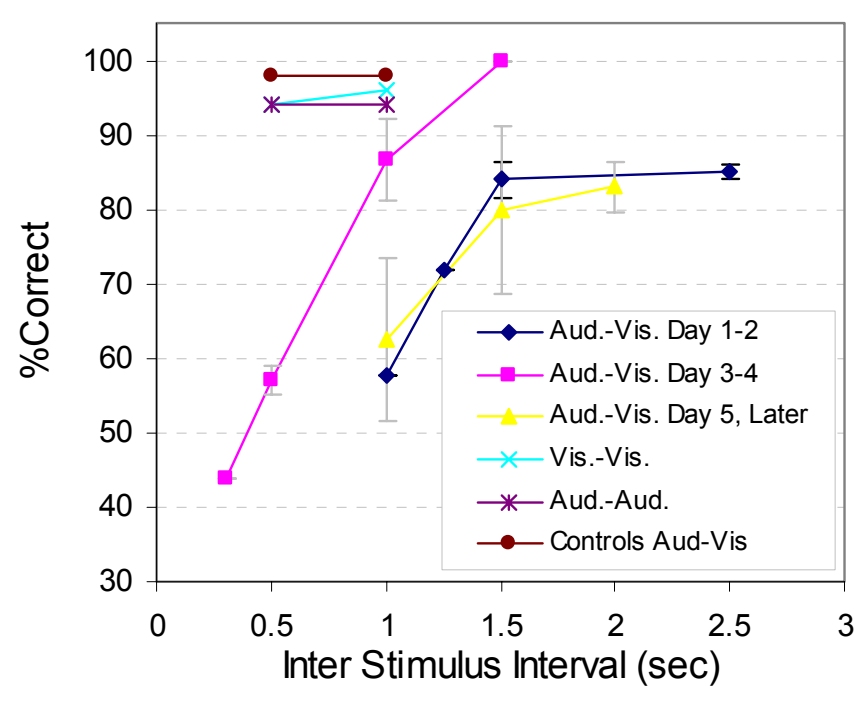

(a)

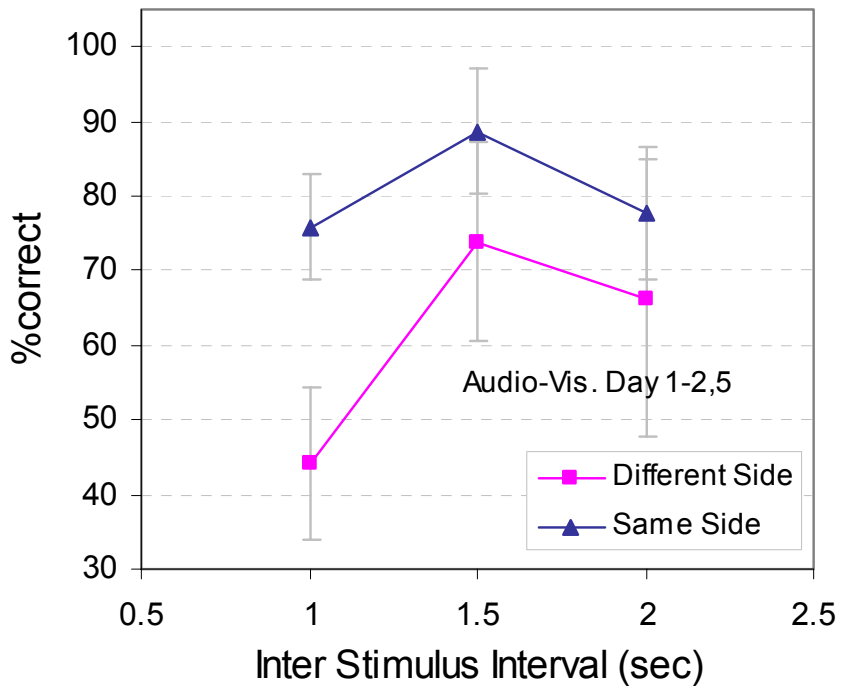

(b)

Figure 3 - Results for the cross-modal auditory-visual extinction in space and time experiment (2). Percentage correct upon judging the same-different stimulus side is plotted as a function of the temporal interval between the onsets of the sound and the flash. (a) Performances from separate testing sessions are plotted separately. Performance is better for longer SOA and shows the effect of practice over closely spaced testing sessions. (b) - data from days 1, 2, 5 are re-plotted separately for the same and different-side stimuli. Performance is significantly better for the same side, compared with different sides. 


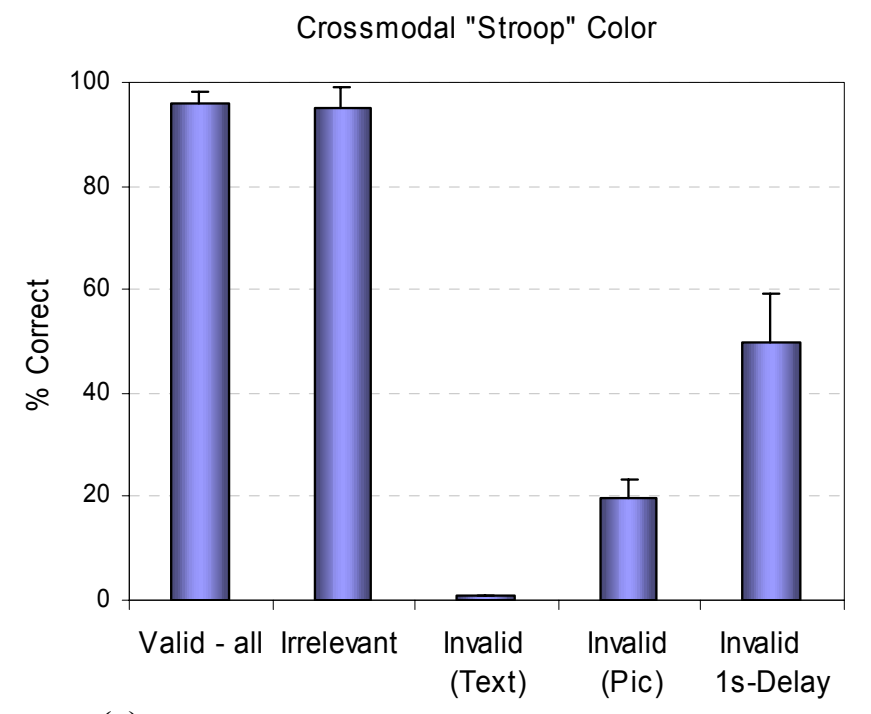

(a)

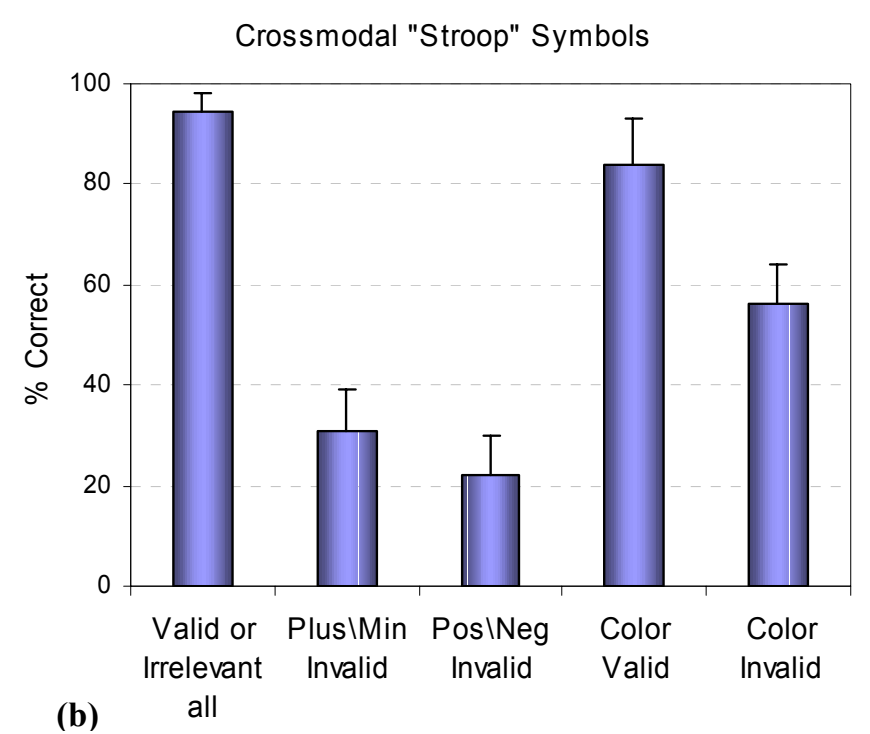

Figure 4 - Results for the cross-modal Stroop experiment (3). Conditions were mixed in randomly ordered trials, and performance (\% correct) is plotted for each condition. In (a) the stimulus was a color patch accompanied by a congruent or incongruent word ("red" or "blue"), or by an irrelevant word ("cat" or "dog"), noise, or silence. All congruent and irrelevant sounds were accumulated (the two left-most bars). The two middle bars show low performance for the incongruent sound, with different performance according to the type of response (see text). The rightmost bar shows that when the patch and the sound were separated by $1 \mathrm{~s}$, performance for invalid sound improved. Panel (b) shows the results of a similar experiment done with symbols (+/-) with congruent, incongruent, and irrelevant sounds (see text). The two rightward bars show that when the symbols were plotted in color and the sounds were color names, performance decreased, especially when the colors were inconsistent (rightmost bar). 
Dual Color/Form Task

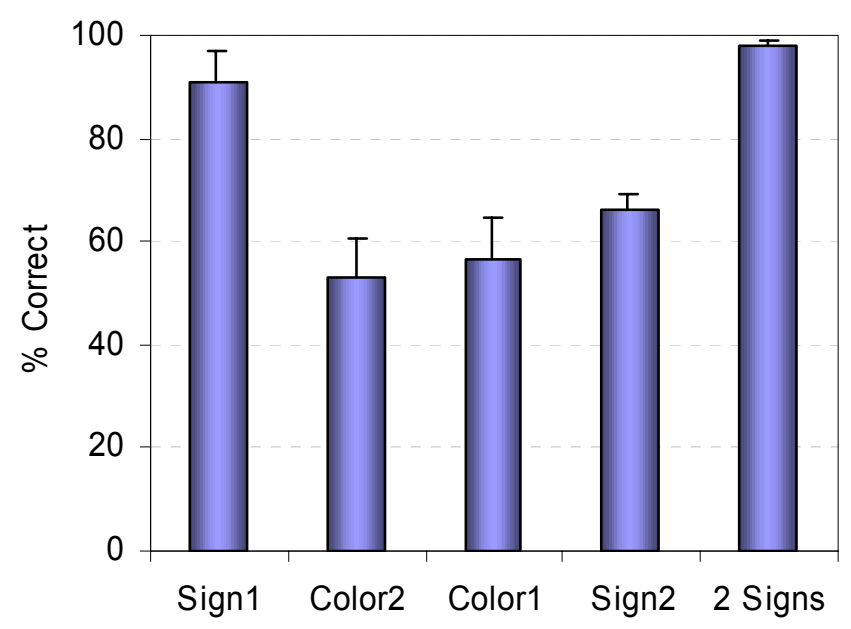

(a)

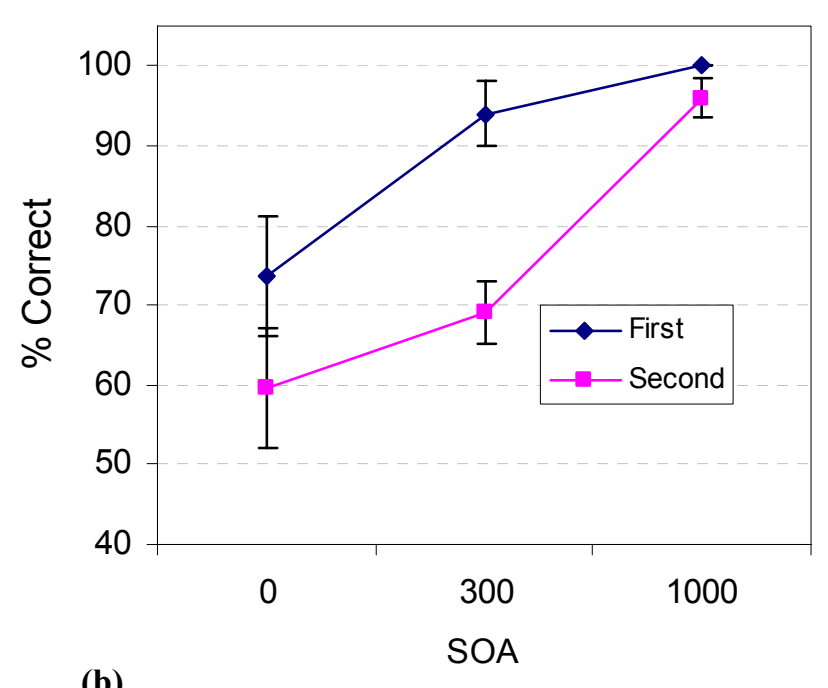

(b)

Dual Color/Form Task - Timing Effect

Figure 5 - Results for the cross-channel color-form experiment (4). A yellow symbol $(+/-)$ and a color (red/blue) patch were briefly presented and the task was to report both in sequence. In (a) performance for color and symbol is plotted, with separate bars for each order of reporting (color first (color1) or sign first (sign1), \&c.). The right-most bar shows performance for the control condition in which 2 symbols had to be identified. When T.M. had to report both color and sign, his performance approached chance level, especially for the color. In (b) the onsets of the symbol and the color patch were separated by $300 \mathrm{~ms}$ and $1 \mathrm{~s}$. Though performance is better for the first item reported (blue plot), both reports reach $100 \%$ correct only with $1 \mathrm{~s}$ SOA. 